\title{
Vitamin B12 Screening in Cervical Spine Surgery Patients
}

\author{
Abdullah Tolaymat ${ }^{1}$, Moataz Abbara ${ }^{1}$, M. Sami Walid ${ }^{2}$, Mohammed Ajjan ${ }^{1}$, Joe Sam Robinson Jr. ${ }^{1}$ \\ ${ }^{1}$ Georgia Neurosurgical Institute; ${ }^{2}$ Medical Center of Central Georgia. \\ Email: tolaymat84@gmail.com
}

Received August $26^{\text {th }}, 2010$; revised September $13^{\text {th }}, 2010$; accepted October $20^{\text {th }}, 2010$.

\begin{abstract}
Introduction: Vitamin B12 is very vital for the nervous system. Its deficiency can manifest with neurological symptoms like pain and paresthesias and in severe cases may cause not completely restorable neurological damage, especially in elderly patients. Methods: The charts of 702 patients who underwent cervical spine surgery retrospectively reviewed and data collected. All patients were preoperatively seen by an internist who ordered Vit B12 levels for some of them. We used two thresholds for the diagnosis of vitamin B12 deficiency, 200 and $300 \mathrm{pg} / \mathrm{mL}$ as recommended by Yao et al. 1992. Data were also collected on gender, payor status, myelopathy, hemoglobin level, corpuscular parameters and glycosylated hemoglobin level $(H b A 1 c)$. Vitamin B12 levels were compared between patients with HbAlc levels $\geq 6.1 \%$ and $<6.1 \%$. Differences between patients $\geq$ and $<$ than the median age were studied. The median age of the patient cohort was 52. Results: Hemoglobin level was recorded for 659 patients. Vit B12 levels were ordered for 291 patients only. Overall, 13.7\% had decreased hemoglobin level (anemia), 30.2\% had decreased Vit B12 levels by the 300 threshold, $6.9 \%$ had decreased Vit B12 levels by the 200 threshold, $6.3 \%$ decreased MCV (microcytosis), and $2.8 \%$ increased MCV (macrocytosis). Only four patients ( $0.7 \%$ ), of whom older than 52, had decreased hemoglobin level and increased MCV (macrocytic anemia) and one patient (0.4\%), who was also older than 52, had decreased hemoglobin level, increased MCV and Vit B12 level $<200 \mathrm{pg} / \mathrm{mL}$ (macrocytic anemia duo to Vit B12 deficiency). Dividing the patient sample into three age groups, $<40,40-59$, and $\geq 60$ years, we investigated the trend of cobalamin deficiency by age and found an increase in cobalamin deficiency after 40 from $0 \%$ to $7.4 \%$ (200 threshold). Uninsured patients (25\%) using the $200 \mathrm{pg} / \mathrm{mL}$ threshold and workers' compensation (54.5\%) and uninsured patients $(50.0 \%)$ had the highest rate of $V i t$ B12 deficiency using the $300 \mathrm{pg} / \mathrm{mL}$ threshold. The few patients with macrocytic anemia $(N=4)$ and macrocytic anemia due to Vit $B 12$ deficiency $(N=1)$ had health coverage. Conclusion: Vit B12 deficiency in cervical spine surgery patients may not necessarily mean macrocytic anemia but may precede macrocytic anemia. Therefore, Vit B12 deficiency screening on the preoperative visit is warranted especially in uninsured or older patients or both. Preoperative treatment may be indicated and correlation with postoperative outcome is suggested for future research.
\end{abstract}

Keywords: Vitamin B12, Cobalamin, Cervical Spine Surgery

\section{Introduction}

Vitamin B12 (cobalamin) is very vital for the nervous system and for the hematogenesis. It can be found in animal products, including fish, meat, poultry, eggs, milk products and fortified breakfast cereals. Vitamin B12 deficiency is thought to be common, especially in the elderly [1]. Only $20 \%$ of patients are younger than age 50 ; most are over 60 [2]. Vitamin $\mathrm{B}_{12}$ absorption decreases greatly in the presence of atrophic gastritis, which is more common in the elderly. Epidemiological studies show a prevalence of cobalamin deficiency of around $20 \%$ (between $5 \%-60 \%$, depending on the definition of cobalamin deficiency used in the study) in the general population of industrialized nations [3]. Vitamin B12 deficiency is also frequently encountered among vegetarians and vegans who do not take B12 supplements [4]. Patients with diabetes type II have also a higher prevalence of Vitamin B12 deficiency. A study by Pflipsen $\mathrm{MC}$, et al., found a prevalence of $22 \%$ among type II diabetes patients [5]. Another study found that $10 \%$ $30 \%$ of patients on metformin develop a vitamin B12 deficiency [6].

\section{Methods}

The charts of 702 patients who underwent cervical spine 
surgery between 2006 and 2008 were retrospectively reviewed and data collected. Our primary aim was to determine the extent of B12 deficiency in the cervical spine surgery population. Our secondary aim was to assess the efficacy of screening for B12 deficiency preoperatively which would improve the overall outcome of surgery if the deficiency is treated.

All patients were preoperatively seen by an internist who ordered Vit B12 levels for some of them. We used two thresholds for the diagnosis of vitamin B12 deficiency, 200 and $300 \mathrm{pg} / \mathrm{mL}$ as recommended by Yao et al. 1992 [7].

The related following parameters were also collected:

- Hemoglobin (HBG) level which was considered low if less than 13.5 grams per deciliter (135 grams per liter) for men and less than 2 grams per deciliter (120 grams per liter) for women.

- Normal mean corpuscular volume (MCV): 80 TO 100 femtoliter.

- Normal mean corpuscular hemoglobin (MCH): 27 to 31 picograms/cell.

- Normal mean corpuscular hemoglobin concentration (MCHC): 32 to 36 grams/deciliter.

Data were also collected on gender, payor status, myelopathy and glycosylated hemoglobin level (HbAlc). Vitamin B12 levels were compared between patients with $\mathrm{HbAlc}$ levels $\geq 6.1 \%$ and $<6.1 \%$. Differences between patients $\geq$ and $<$ than the median age were studied. The median age of the patient cohort was 52 .

\section{Results}

Hemoglobin level was recorded for 659 patients. Vit B12 levels were ordered for 291 patients only. Overall, 13.7\% had decreased hemoglobin level (anemia), 30.2\% had decreased Vit B12 levels by the 300 threshold, $6.9 \%$ had decreased Vit B12 levels by the 200 threshold, $6.3 \%$ decreased MCV (microcytosis), and $2.8 \%$ increased MCV (macrocytosis).

The average age of cobalamin deficient patients (200 threshold) was 53.6 years versus 55.3 years for cobalamin normal patients. Using the 300 threshold, the results were the opposite, 51.4 years for cobalamin deficient and 54.7 for cobalamin normal patients.

Only four patients $(0.7 \%)$ had decreased hemoglobin level and increased MCV (macrocytic anemia) and one patient $(0.4 \%)$ had decreased hemoglobin level, increased MCV and Vit B12 level $<200 \mathrm{pg} / \mathrm{mL}$ (macrocytic anemia duo to Vit B12 deficiency).

Anemia was more common (16.8 vs. $10.2 \%)$ in the older half of the patient cohort $(\mathrm{P}<0.05)$. Three of the four patients with macrocytic anemia were older than 52 and the only patient with macrocytic anemia due to Vit B12 deficiency was older than 52.
No significant differences between the genders or between patients with or without myelopathy regarding the above variables were noted. Paradoxically, significant differences in the percentages of vitamin B12 deficiency $(<300 \mathrm{pg} / \mathrm{mL}$ threshold) levels were noted between patients with $\mathrm{HbA} 1 \mathrm{c}$ levels $\geq 6.1 \%$ and $<6.1 \%$ (33.5\% vs. $20.0 \%, \mathrm{P}<0.05)$ and those $\geq$ and $<$ than the median age ( $38.6 \%$ vs. $23.8 \%, \mathrm{P}<0.01)$ in favor of younger patients and those with normal HbAlc levels (Table 1). As expected, anemia (decreased hemoglobin level) was more common in older patients and those with increased HbAlc level.

Dividing the patient sample into three age groups, $<40$, 40 - 59 , and $\geq 60$ years, we investigated the trend of cobalamin deficiency by age and found an increase in cobalamin deficiency after 40 from $0 \%$ to $7.4 \%$ (Figure 1).

Uninsured patients (25\%) using the $200 \mathrm{pg} / \mathrm{mL}$ threshold and workers' compensation (54.5\%) and uninsured patients $(50.0 \%)$ had the highest rate of Vit B12 deficiency using the $300 \mathrm{pg} / \mathrm{mL}$ threshold (Figure 2). The few patients with macrocytic anemia $(N=4)$ and macrocytic anemia due to Vit B12 deficiency $(N=1)$ had health coverage.

\section{Discussion}

Many studies conclude that the levels of serum vitamin B-12 concentrations currently considered normal in the United States may be too low and should be reassessed. The lower limit of $200 \mathrm{pg} / \mathrm{mL}$ is based on the level that causes abnormalities in the blood (pernicious anemia) [8]. In contrast, the lower limit in Japan and some European countries is $500-550 \mathrm{pg} / \mathrm{mL}$ [8]. A study by Yao et al. 1992 recommends that the lower limit of the normal range for cobalamin should be increased to $300 \mathrm{pg} / \mathrm{mL}$ [7].

One Swedish study of 368 men and women aged 75 years and older concluded that routine screening for vitamin B-12 deficiency is justified in older patients [9]. We have used two thresholds in our study and found somewhat paradoxical results with the higher threshold in older patients and patients with increased $\mathrm{HbAlc}$, contrary to some previous reports $[5,6]$. We thus recommend abiding by the traditional threshold for the diagnosis of Vit B12 deficiency, $200 \mathrm{pg} / \mathrm{mL}$.

About $40 \%$ of all patients with B12 deficiency complain of neurological symptoms, which are often the first manifestation of the disease, usually, a combination of myelopathy and peripheral neuropathy [2]. The most common symptom attributable to neuropathy is burning and painful sensations in the hands and feet known as acroparesthesia $[2,10]$. In the literature, there are case reports of patients who underwent spine surgery who were later found to have Vit B12 deficiency [11,12]. In 
Table 1. Crosstabulation of catgorical variables.

\begin{tabular}{|c|c|c|c|c|c|c|c|c|c|}
\hline & & \multicolumn{4}{|c|}{ HbA1cCoded } & \multicolumn{4}{|c|}{ AgeCategorical } \\
\hline & & \multicolumn{2}{|c|}{$<6.1 \%$} & \multicolumn{2}{|c|}{$\geq 6.1 \%$} & \multicolumn{2}{|c|}{$<52$ Years } & \multicolumn{2}{|c|}{$\geq 52$ years } \\
\hline & & $\mathrm{N}$ & $\mathrm{N} \%$ & $\mathrm{~N}$ & $\mathrm{~N} \%$ & $\mathrm{~N}$ & $\mathrm{~N} \%$ & $\mathrm{~N}$ & $\mathrm{~N} \%$ \\
\hline \multirow{2}{*}{ Vit B12 Deficiency (200 Threshold) } & Normal & 187 & $92.1 \%$ & 72 & $96.0 \%$ & 120 & $94.5 \%$ & 151 & $92.1 \%$ \\
\hline & Deficient & 16 & $7.9 \%$ & 3 & $4.0 \%$ & 7 & $5.5 \%$ & 13 & $7.9 \%$ \\
\hline \multirow{2}{*}{ Vit B12 Deficiency (300 Threshold) } & Normal & 135 & $66.5 \%$ & 60 & $80.0 \%$ & 78 & $61.4 \%$ & 125 & $76.2 \%$ \\
\hline & Deficient & 68 & $33.5 \%$ & 15 & $20.0 \%$ & 49 & $38.6 \%$ & 39 & $23.8 \%$ \\
\hline \multirow{2}{*}{ HBG } & Normal & 362 & $89.8 \%$ & 119 & $81.5 \%$ & 282 & $89.8 \%$ & 287 & $83.2 \%$ \\
\hline & Decreased & 41 & $10.2 \%$ & 27 & $18.5 \%$ & 32 & $10.2 \%$ & 58 & $16.8 \%$ \\
\hline \multirow{3}{*}{$\mathrm{MCV}$} & Normal & 344 & $92.0 \%$ & 128 & $92.8 \%$ & 269 & $91.5 \%$ & 279 & $90.3 \%$ \\
\hline & Decreased & 17 & $4.5 \%$ & 8 & $5.8 \%$ & 16 & $5.4 \%$ & 22 & $7.1 \%$ \\
\hline & Increased & 13 & $3.5 \%$ & 2 & $1.4 \%$ & 9 & $3.1 \%$ & 8 & $2.6 \%$ \\
\hline \multirow{2}{*}{$\mathrm{MCH}$} & Normal & 351 & $96.2 \%$ & 126 & $93.3 \%$ & 273 & $95.1 \%$ & 280 & $94.0 \%$ \\
\hline & Decreased & 14 & $3.8 \%$ & 9 & $6.7 \%$ & 14 & $4.9 \%$ & 18 & $6.0 \%$ \\
\hline \multirow{2}{*}{$\mathrm{MCHC}$} & Normal & 361 & $98.9 \%$ & 130 & $96.3 \%$ & 284 & $99.0 \%$ & 288 & $96.6 \%$ \\
\hline & Decreased & 4 & $1.1 \%$ & 5 & $3.7 \%$ & 3 & $1.0 \%$ & 10 & $3.4 \%$ \\
\hline
\end{tabular}

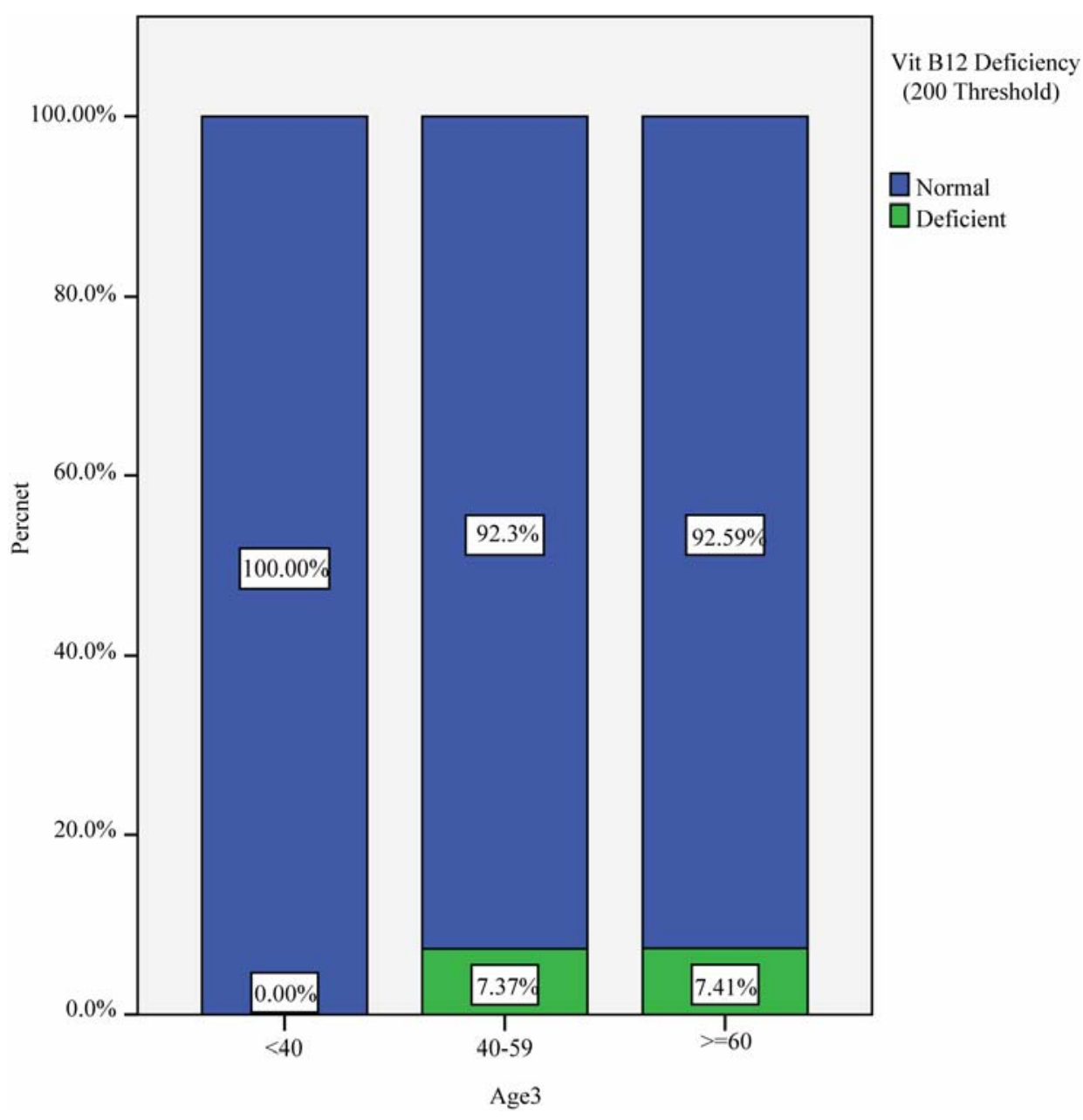

Figure 1. Vitamin B12 deficiency prevalence per age group using the $200 \mathrm{pg} / \mathrm{mL}$ threshold. 


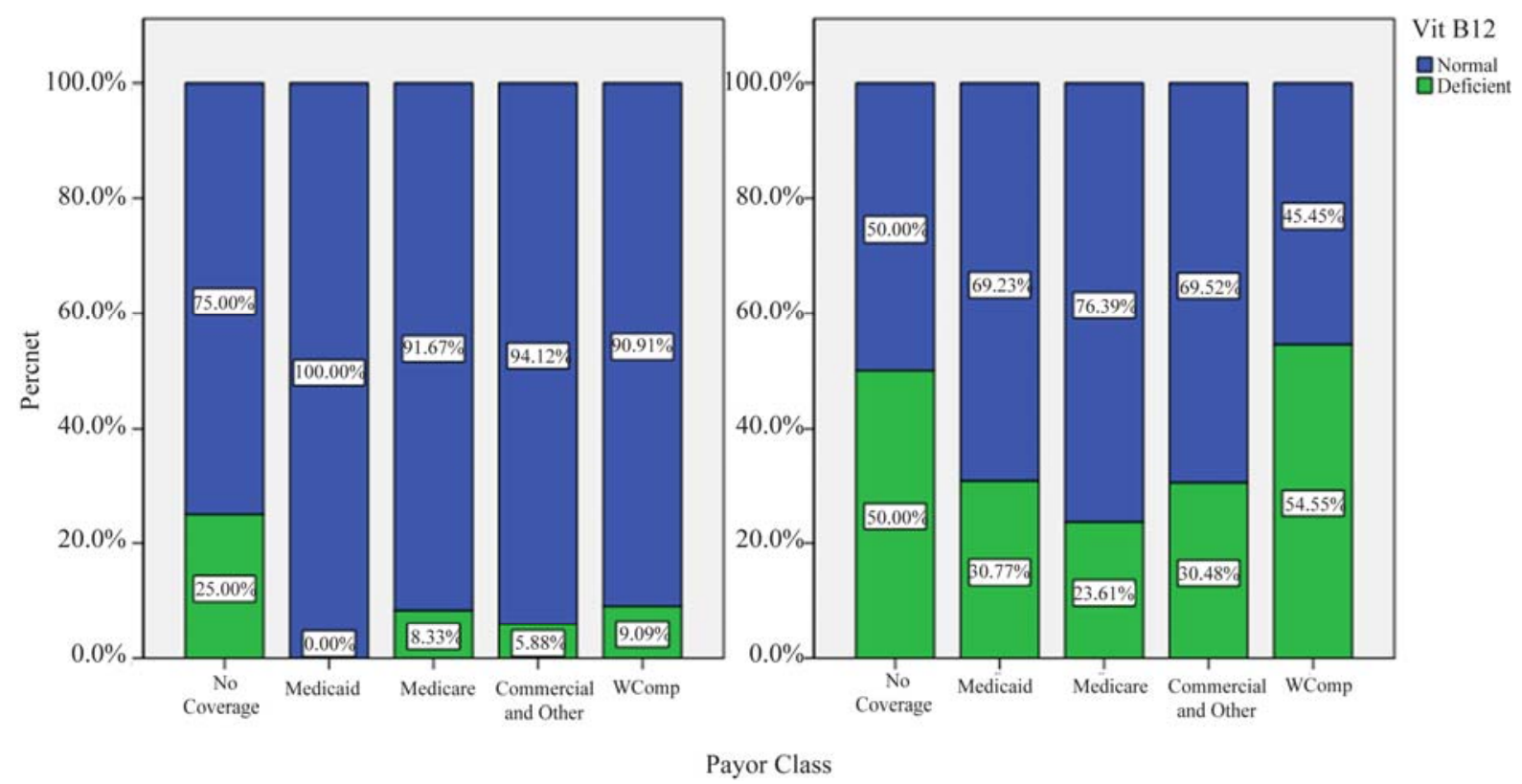

Figure 2. Vitamin B12 deficiency prevalence per payor class using the $200 \mathrm{pg} / \mathrm{mL}$ threshold (left) and the $300 \mathrm{pg} / \mathrm{mL}$ threshold (right).

patients with neurologic signs, only about $20 \%$ show severe anemia [2]. Both the hematocrit and mean corpuscular volume may be normal [2].

This paper underscores the importance of considering vitamin B12 deficiency in the differential diagnosis of patients whose presentations seem to indicate only a surgically correctable spinal cord disorder. We recommend considering B12 test as a routine test for all patients with age 50 and over, for patients who complain of peripheral sensation abnormalities and for those who present for spine surgery, especially uninsured and workers compensation patients. Larger studies are needed to clarify the true prevalence of cobalamin deficiency in spine surgery candidates belonging to different payor classes and the impact of such screening on spinal surgery outcome. In the meanwhile, because the window of opportunity for treatment and reversal of neurologic complications is short, usually measured in months, Dharmarajan et al. 2003 recommend starting screening for Vit B12 deficiency at age 50, then every five years until age 65 and afterwards annually [13].

\section{REFERENCES}

[1] S. Loikas, P. Koskinen, et al., "Vitamin B12 Deficiency in the Aged: A Population-Based Study," Age and Ageing, Vol. 36, No. 2, 2007, pp. 177-183. doi:10.1093/ageing/afl150

[2] L. P. Rowland and B. P. Worrall, "Nutritional Disorders: Vitamin B12 Deficiency, Malabsorption, and Malnutrition," 10th Edition, Lippincott Williams \& Wilkins Pub- lishers, Broadway, 2000.

[3] E. Andrès, et al., "Vitamin B12 Deficiency in Elderly Patients," Canadian Medical Association Journal, Vol. 171, No. 3, 2004, pp. 251-259. doi:10.1503/cmaj.1031155

[4] A. Dong and S. C. Scott, "Serum Vitamin B12 and Blood Cell Values in Vegetarian," Annals of Nutrition and Metabolism, Vol. 26, No. 4, 1982, pp. 209-216. doi: $10.1159 / 000176565$

[5] M. C. Pflipsen, et al., "The Prevalence of Vitamin B12 Deficiency in Patients with Type 2 Diabetes: A Cross-Sectional Study," The Journal of the American Board of Family Medicine, Vol. 22, No. 5, 2009, pp. 528-534. doi:10.3122/jabfm.2009.05.090044

[6] M. A. Gilligan, "Metformin and Vitamin B12 Deficiency," Archives of Internal Medicine, Vol. 162, No. 4, 2002, pp. 484-485. doi:10.1001/archinte.162.4.484

[7] Y. Yao, et al., "Prevalence of Vitamin B12 Deficiency among Geriatric Outpatients," The Journal of Family Practice, Vol. 35, No. 5, 1992, pp. 524-528.

[8] M. Goodman, et al., "Are U.S. Lower Normal B-12 Limits Too Low?" Journal of the American Geriatrics Society, Vol. 44, No. 10, 1996, pp. 1274-1275.

[9] R. Eggertsen, et al., "Prevalence and Diagnosis of Cobalamin Deficiency in Older People," Journal of the Amer-ican Geriatrics Society, Vol. 44, No. 10, 1996, pp. 1273- 1274.

[10] E. V. Healton, et al., "Neurologic Aspects of Cobalamin Deficiency," Medicine, Vol. 70, No. 4, 1991, pp. 229244. doi:10.1097/00005792-199107000-00001

[11] K. D. Herr, et al., "Acute Psychosis in a Patient with Vitamin B12 Deficiency and Coincident Cervical Stenosis," 
Psychosomatics, Vol. 43, No. 3, 2002, pp. 234-236. doi:10.1176/appi.psy.43.3.234

[12] R. Smith, "Sudden Onset of Psychosis in Association with Vitamin B12 Deficiency," British Medical Journal,
Vol. 3, No. 5556, 1967, p. 34. doi:10.1136/bmj.3.5556.34

[13] T. S. Dharmarajan, et al., "Vitamin B12 Deficiency. Recognizing Subtle Symptoms in Older Adults," Geriatrics, Vol. 58, No. 3, 2003, pp. 37-38. 